\title{
Position-sensitive SiPM detector for separation of Cherenkov and fluorescent light of EAS
}

D. Chernov ${ }^{a}$, E. Bonvech ${ }^{a}$, T. Dzhatdoev ${ }^{a}$, Mir. Finger $^{b c}$, Mich. Finger $^{b c}$, V. Galkin ${ }^{d a}$, G. Garipov ${ }^{a}$, V. Kozhin ${ }^{a}$, D. Podgrudkov ${ }^{* d a}$, A. Skurikhin ${ }^{a}$.

${ }^{a}$ Skobeltsyn Institute of Nuclear Physics, Lomonosov Moscow State University, Leninskie gory 1-2, 119991, Moscow, Russia

${ }^{b}$ Faculty of Mathematics and Physics, Charles University, Ovocny trh 3-5, 116 36, Prague 1, Czech Republic

c Joint Institute for Nuclear Research, Joliot-Curie 6, 141980 Dubna, Moscow region, Russia

${ }^{d}$ Faculty of Physics, Lomonosov Moscow State University, Leninskie gory 1-2, 119991, Moscow, Russia

E-mail: chredec1.sinp.msu.ru

\begin{abstract}
A detector is designed for ground and space based optical experiments for high and ultrahigh energy cosmic ray studies. In this paper a separation method based on the simultaneous light recording from one 'optical pixel' by two or two pairs of silicon photomultipliers (SiPMs) is proposed. The first SiPM detects incoming light flux in the wavelength band of its maximal sensitivity. The second SiPM detects light through an optical UV filter for fluorescent light (FL). If the sensitivity characteristics of the SiPMs, the absorption characteristics of the filter elements, and the spectra of fluorescent and Cherenkov light (CL) are known, one can calculate the contribution of each component to the total light flux. Upon the completion of this work it will be possible to separate fluorescent and Cherenkov light at the stage of on-board primary processing of recorded data. This method will increase methodological accuracy of fluorescent light measurements and thus significantly reduce the impact of simulations for primary cosmic ray characteristics reconstruction.
\end{abstract}

35th International Cosmic Ray Conference - ICRC2017

10-20 July, 2017

Bexco, Busan, Korea

${ }^{*}$ Speaker. 

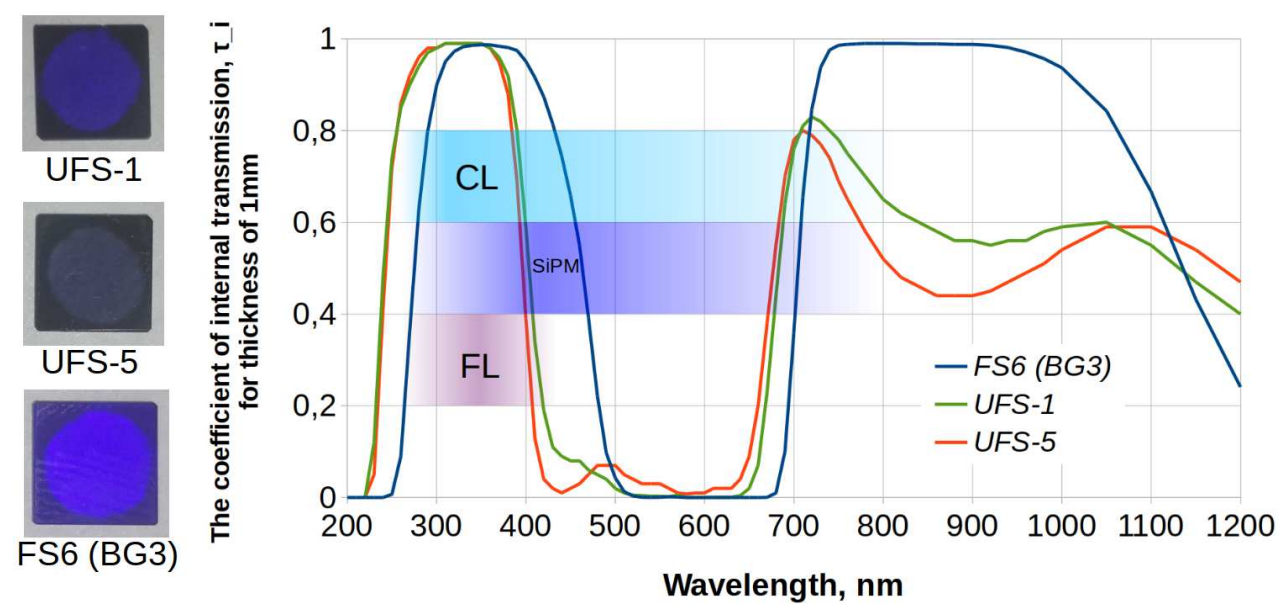

Figure 1: On the left: the light filters photo over a white LED. On the right: Spectral characteristics of light filters FS6 (BG3 analogue), UFS-1, UFS-5. Color bars indicate the spectral ranges of the CL and FL and of the SiPM sensitivity region.

\section{Introduction}

Direct and scattered Cherenkov light (CL) is one of the relevant contributions to the uncertainty of the measured flux of fluorescent light (FL) from extensive air showers (EAS). The impact of the backscattered CL was noted in the detector response modelling for the JEM-EUSO project [1]. The ground based FL detectors of the Pierre Auger Observatory also collect CL and a correction is applied to account for that during the cascade curve reconstruction [2],[3]. In the TALE experiment it was made one of the most successful to date attempt to isolate CL using the angular distribution of light at the detector [4]. The main idea of this method is that CL comes to the detector from a narrow angle (about few degrees) and the FL has a much wider angular distribution. However, the $\mathrm{CL} / \mathrm{FL}$ ratio was not measured in the experiment but was estimated using the modelled results for the known EAS zenith angle and distance to the shower core. Furthermore, data with expected FL fraction lower than $80 \%$ was excluded from the following analysis. This was partially due to the strong model dependences in EAS development, especially in cases where CL and FL fluxes were comparable. Therefore, the problem of a reliable CL and FL separation is relevant for the better high energy EAS registration and primary particle parameters estimation.

\section{Idea of the method}

As was said above, the aim of this study is the enhancement of the precision of EAS optical components registration and primary particle parameters (mass and energy) estimation. The study includes both theoretical approaches to the problem and the possible electronics design for the ground, airborne and space-based detectors.

The main idea of the proposed method uses the simultaneous registration of incident light by two or more photodetectors, one of which collects all photons and the other or others collect photons after a specific filter. In other words, this method utilizes the same logic as a common photocamera. For this method to be effective, the photodetector size should be $\sim 2$ times smaller 
than the optical part resolution. In this case the measurement results are independent from the size or shape of the registered image. This condition is well met by the silicon photomultipliers since their sizes are typically smaller than those of common photomultiplier tubes (PMT) and are about $1 \times 1 \mathrm{~mm}$ to $6 \times 6 \mathrm{~mm}$. With the known CL and FL spectra, filters and photodetectors spectral characteristics, the contribution of each optical component can be reconstructed. For example, the UFS-1 filter transmits up to $80 \%$ of FL in the $250-370 \mathrm{~nm}$ range (see figure 3), but does not transmit more than $90 \%$ of the CL 'tail' in the 420-650 nm range. UFS-5 and FS6 (the BG3 analogue) have close characteristics to the ones described above. In other words, the method uses the fact that the CL spectrum is much wider than the FL one so that the appropriately selected filter would allow to separate CL and FL based on the pixel signals difference.

\section{Development of the detector}

In most of the present-day EAS optical components detectors the common PMTs are used, but lately SiPMs became of some use. SiPMs have sensitivity comparable to PMT's one, but are more compact, lighter and do not require high voltage power supplies. It is expected that further development of SiPMs will lower their cost with simultaneous increase in their sensitivity. For the current study the SensL SiPMs with $\sim 6 \times 6 \mathrm{~mm}$ [5] sensitive area were chosen. The differential SiPM's 'fast output' with good time resolution ( $\sim 5 \mathrm{~ns})$ allows to use them in the photon counting mode. This mode allows to forgo the use of complex and power consuming analog-to-digital converters (ADC). Instead of ADCs the output of each SiPM is routed to the analog voltage comparators with digital trigger counters. The measuring channel can be implemented using field-programmable gate array chip (FPGA). The 5 ns sampling frequency exclude the photons miscount (both a miss and double count) since the SiPM signal width is also around $5 \mathrm{~ns}$. The comparator trigger voltage is set to the voltage of a single photoelectron that is individual to each SiPM. Therefore the data acquisition system records signal not in an arbitrary units but directly in the photoelectrons that greatly simplifies the following analysis. However, the SiPMs have significant dark current (noise) in common conditions. But the level of that noise is comparable in most cases to the starlight background hence the impact of the SiPM dark current on the overall fluctuations in real experiment will be around additional $\sim 40 \%$ over the ideal detector. Moreover, low temperatures (around $-20^{\circ} \mathrm{C}$ and below) decrease the SiPM dark current 10-20 times (the rate is about $\sim 2$ times over each $10^{\circ} \mathrm{C}$ ).

For the studies the MicroFC-SMTPA-60035 SiPM was selected and its quantum efficiency was measured. The SiPM voltage was set to $29.20 \mathrm{~V}$ (24.5 V — the main voltage and 4.7V overvoltage as per specification). The measurements were done using the Hamamatsu L11494-430 calibrated light source [9]. Three measuring series were recorded using an 2 Gs/s oscillograph:

1. with both the SiPM power suppliers and the light source switched off;

2. with SiPM powered, but the light source switched off;

3. with both the SiPM power suppliers and the light source switched on.

The recorded series with the SiPM switched off allowed to measure the amplifier noise level. The same was repeated for two other series. But both of the other series show distinct peaks 


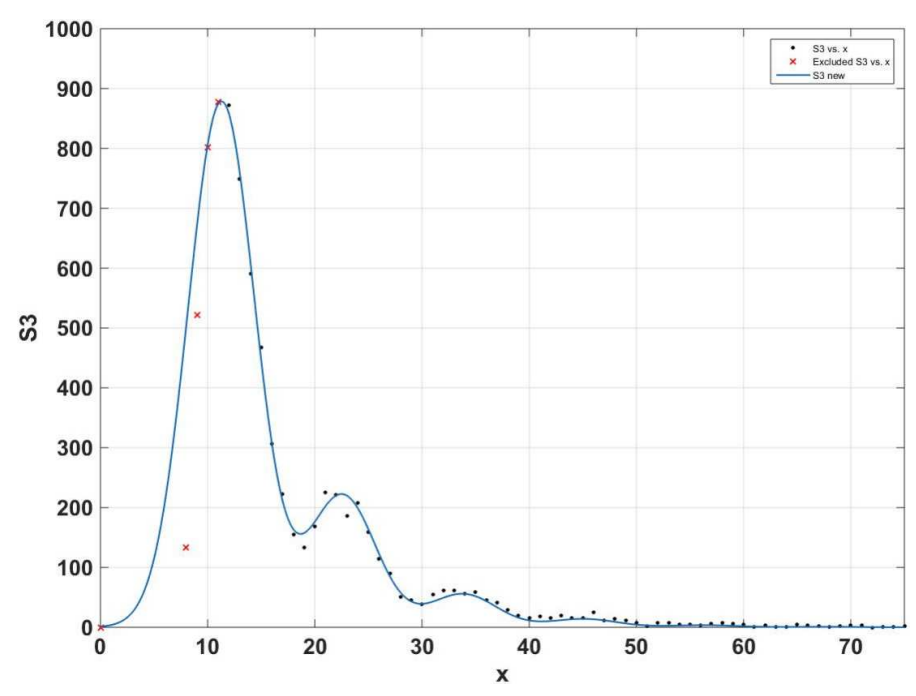

Figure 2: The amplitudes of the pulses recorded from the SiPM output with the light source switched on (the amplifier noise is subtracted from the data) is shown by the dots. The curve represent the fit using sum of multiple Gauss distributions.

from multiple or single photoelectrons. The Hamamatsu L11494-430 light source, according to the previous measurements (see [6] for details), gives random flux of single photons with low portion of pairs (none were reliably detected during the previous measurements). The multiple photoelectron peaks (see figure 2) in the recorded data from this SiPM are caused by the induced triggering of the neighboring elementary cells. This conclusion is in agreement with the exponential decay of the distribution peaks amplitude in the figure 2. In case of multiple photons being emitted from the light source their number should follow the Poisson distribution.

After the noise subtraction the amplitude histogram was fitted to correctly estimate the total number of registered photoelectrons in assumption that each subsequent peak to the right is the 'shadow' of the single photoelectron peak. Each photon-produced electron in an elementary cell has some chance $p$ to cause the neighbouring elementary cell to produce another electron. This probability is fixed and does not depend on the number of triggered elementary cells (in assumption that the total number of triggered cells in much less that the total number of cells). In this assumption the expected amplitude distribution will be $N(x)=\sum_{n=1}^{\infty} p^{n-1} N\left(n x_{0}, \sigma\right)$, where $N\left(x_{0}, \sigma\right)-$ normal distribution with average $x_{0}$ and dispersion $\sigma$. This theoretical curve fits the data better than the one in assumption of multiple photons being emitted from the light source that should give much lower numbers of high photon multiplicity cases.

The data recorded in the second measuring series (with SiPM powered on but the light source turned off) allowed to measure the SiPM dark current equal to the $1750 \pm 40$ photoelectrons in $600 \mu \mathrm{s}$ (approximately $2.917 \mathrm{MHz}$ ). The multiple photoelectron peaks were also observed in this series. The measurements with light source turned on gave $6870 \pm 150$ photoelectrons in $2 \mathrm{~ms}$ (3.435 MHz). In this case the total number of photoelectrons include both the dark current and the actual photoelectrons. Hence the frequency of the photoelectrons from light source photons was about $478 \mathrm{kHz}$. The light source emits about $2.12 \times 10^{6}$ photons/s. The resulting quantum efficiency of the studied SiPM was about $24 \pm 2 \%$. The fill coefficient (the portion of the total 

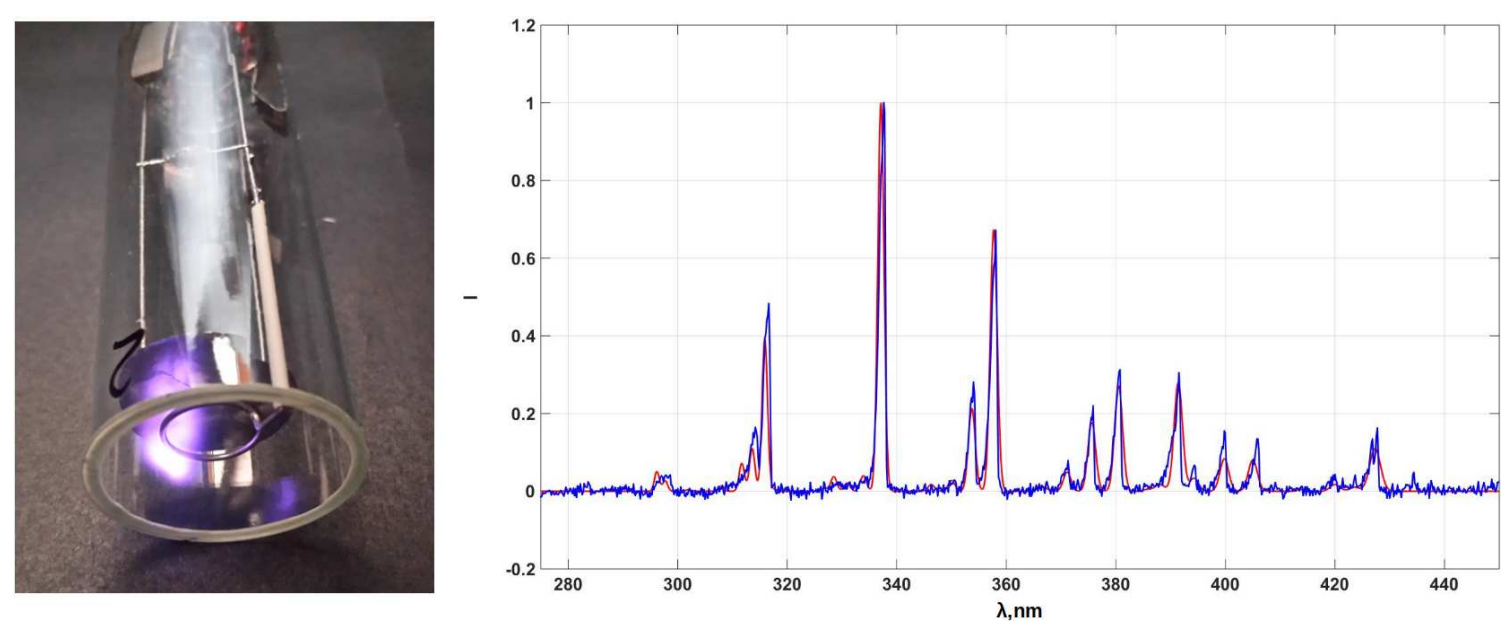

Figure 3: On the left: the FL source photo. On the right: blue curve shows the measured spectrum of the FL source, the red curve presents the FL spectrum from the $293 \mathrm{~K}$ dry air at $800 \mathrm{hPa}$ according to the AIRFLY collaboration data.

area that is sensitive to light) for the SiPM is 0.64 according to the manufacturer specification [5]). Combined this gives quantum efficiency of a single cell about $38 \pm 3 \%$ which is in a good agreement with the manufacturer data.

For the next step of this study (that included the FL-CL separation method efficiency estimation) the test bench was created. For the CL source the $50 \times 15 \times 15 \mathrm{~mm}$ acrylic radiator penetrated by cosmic ray muons was used. Two additional $15 \times 15 \times 15 \mathrm{~mm}$ scintillator detectors set in coincidence mode were used to select only those muons that passed through the whole radiator.

The FL source was designed by request in the 'MELZ FEU' Ltd. [7]. The source was made using standard $30 \mathrm{~mm}$ PMT tube (see figure 3). The output window was made from $\mathrm{MgF}_{2}$ optical glass. For this study two sources were made: one filled with air at $50 \mathrm{hPa}$ (No.1) and $10 \mathrm{hPA}$ (No.2). Stable light emission (glow discharge) appears at 500-600 V voltage between the source's electrodes (with $1 \mathrm{MOhm}$ load resistor). The FL flashes were generated using short voltage pulses between the electrodes. On the figure 3 the measured spectrum from the light source No.2 (10 pHa) is presented. The measure spectrum is in a good agreement with the AIRFLY collaboration data [8] even at different pressure.

Altering the sources (and it is know which source produced a light pulse) the SiPM response was recorded with and without the filters. The aim of the following measurements is in the accurate test of the different optical filters (with different thickness) to achieve best possible CL and FL separation. Preliminary results are expected to be published in the end of 2017.

\section{Modeling and Calculations}

To estimate the efficiency of the proposed CL-FL separation method and its impact on the precision of the EAS primary particle properties estimation, an EAS simulation was performed with account of realistic light scattering and absorption in the atmosphere. Widely used software package for statistical simulation of EAS - CORSIKA [10] - allows to calculate many of the 
characteristics of EAS in detail. It can be used to model the differential distributions of CL, however, the option for modeling fluorescent light is missing. Accounting for the optical properties of the atmosphere in CORSIKA is minimal, it is limited to an approximate registration of absorption. Thus, the goal of realistic reproduction of the optical image of an EAS detector cannot be solved by means of the CORSIKA only. Simulation photons transport in the atmosphere can be made using another widely known program complex — GEANT4 [11],[12],[13] that allows the account of absorption, scattering, reflection, refraction and light generation processes in a variety of environments and to flexibly manage the process of radiation propagation. Combining the power of two complexes gives the opportunity to solve the problem of realistic modeling of the optical image of an EAS in the atmosphere.

The first stage of problem solution requires to obtain the angular distributions of CL and FL from a single high energy EAS in optical detector installed at sea level. Based on the CORSIKA and GEANT4 capabilities and accessible information about the optical properties of the atmosphere the solution should be divided into three stages:

1. The generation of EAS in CORSIKA with recording in a file of emission points, directions, times and wavelengths of Cerenkov photons from charged particles. At the same time the energy depositions of the cascade particles in air are accumulated within a certain volumes that includes a major part of the particles. A three-dimensional array of energy depositions is recorded in a separate file.

2. Using file with energy depositions the FL photons are generated isotropic emission. For the downward going photons the points of emission, directions, times and wavelengths were recorded in a separate file.

3. Using GEANT4 the atmosphere model was built that consisted of homogeneous horizontal layers, each with its own density-dependent wavelength refractive index, absorption length and other optical properties. Files of CL and FL photons were used as a source, the light from them was traced to the absorption point, the volume boundary outside the atmosphere or to the detectors. The photon reaching the detector were histogrammed in the directions and wavelengths.

The hybrid scheme described above was implemented. With its help the Cherenkov and fluorescence images of the inclined EAS from $1 \mathrm{PeV}$ primary proton were obtained. The atmosphere extended for $100 \mathrm{~km}$ up from sea level, from North to South and from East to West. The shower axis fell in the centre of the base of the atmosphere cube. The dependence of air density from height corresponded to the standard US atmosphere (same in both CORSIKA and GEANT4). Air cube was divided into 20 horizontal layers with a vertical thickness of $\sim 52 \mathrm{~g} / \mathrm{cm}^{2}$. The refractive index of each layer corresponded to its average density.

A region of atmosphere in which the particles deposited energy represented an inclined elliptical cylinder ( $1 \mathrm{~km}$ circle in a horizontal plane), the axis of which coincided with the axis of the shower. The cylinder was divided by vertical atmospheric layers. The disk layer was divided into the ring along the radius and the sectors in azimuth. The FL photons were emitted from the centres of the three-dimensional cells. The fluorescence yield was set to 10 photons per $1 \mathrm{MeV}$. The spectrum of the emitted photons matched the emission spectrum of nitrogen molecules. The 

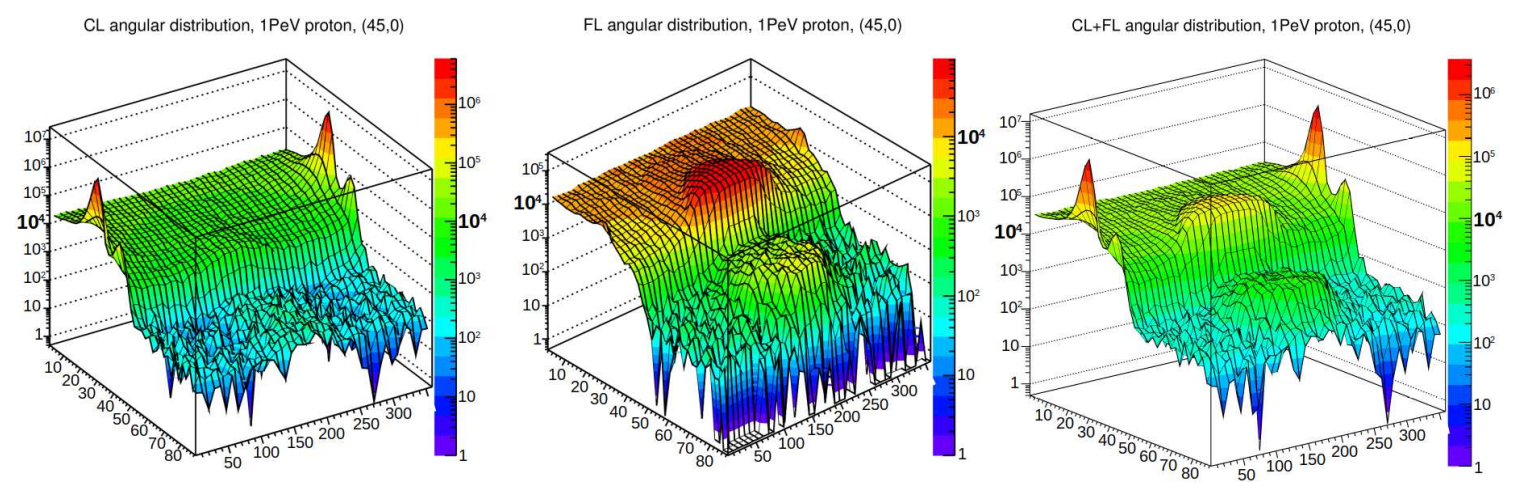

Figure 4: The histograms of CL (left) and FL (middle ) photons numbers from $1 \mathrm{PeV}$ primary proton EAS in polar coordinates. On the right - the sum of both $\mathrm{CL}$ and FL photons distributions. The data is presented for 200-440 nm wavelengths.

GEANT4 traced optical photons in the cube of the atmosphere accounting for absorption (including absorption of atmospheric ozone) and molecular scattering. Tracking a huge number of Cherenkov photons required large computational resources. CORSIKA allows to generate photon in bunches. Our code also generated FL photons in bunches to save computational resources. For both types of photons for the $1 \mathrm{PeV}$ shower 100 photon bunches were used.

On figures 4 the CL and FL images (angular distributions) are presented for the $0.25 \mathrm{~km}^{2}$ detector positioned at $(-0.45 ; 0.00) \mathrm{km}$ point. The shower had $45^{\circ}$ zenith and $0^{\circ}$ azimuth angle, e.g. its axis is just above the center of the detector. The detector's field of view roughly coincided with the upper hemisphere. Each cell spans 1 square degree and shows the integral number of photons in the 200-440 nm wavelength region. The figures show that scattered CL gives a significant contribution to the total FL flux.

\section{Conclusions}

To study CL and FL from EAS separation methods the characteristics of SiPM that will be used in future detector were measured; the CL and FL sources were designed and prepared; the first version of the detector response simulation code was created. The study of operation and performance of SiPM samples showed that the average quantum efficiency was $24 \pm 2 \%$ and the quantum efficiency of an elementary cell was $38 \pm 3 \%$. These values are comparable to widely used at present common PMTs. The FL source has a good agreement of spectral characteristics with the world data. The use of this source greatly simplifies the testing method for the separation of CL and FL. CORSIKA-based program allows to estimate the performance of a new proposed system for new ground or space-based experiments. The following new detector modeling will use the obtained results of CL-FL separation accuracy.

\section{Acknowledgments}

This work was funded by Russian Foundation for Basic Research (RFBR) according to the research project No.16-02-00777. 


\section{References}

[1] A. Haungs et al. for JEM-EUSO Collaboration, Physics Goals and Status of JEM-EUSO and its Test Experiments, Journal of Physics: Conference Series 632 (2015) 012092 [arXiv: 1504 . 02593 ].

[2] The Pierre Auger Collaboration, A Study of the Effect of Molecular and Aerosol Conditions in the Atmosphere on Air Fluorescence Measurements at the Pierre Auger Observatory, Astroparticle Physics 33 (2010) 108 [arXiv: 1002 .0366].

[3] M. Unger, B.R. Dawson, R. Engel, F. Schussler, R. Ulrich, Reconstruction of longitudinal profiles of ultra-high energy cosmic ray showers from fluorescence and Cherenkov light measurements, Nucl. Instrum. Methods A588 (2008) 433 [arXiv: 0801 .4309].

[4] T. AbuZayyad for the Telescope Array Collaboration, Cosmic Rays Energy Spectrum observed by the TALE detector using Cerenkov light, in proceedings of International Cosmic Ray Conference, POS ( ICRC2015) 422 (2016).

[5] http://sensl.com/.

[6] R.A. Antonov, E.A. Bonvech, D.V. Chernov, D.A. Podgrudkov, T.M. Roganova, The LED calibration system of the SPHERE-2 detector, Astroparticle Physics 77 (2016) 55.

[7] http://www.melz-feu.ru/.

[8] M. Ave et al. for AIRFLY Collaboration, Measurement of the pressure dependence of air fluorescence emission induced by electrons, Astropart. Phys. 28 (2007) 41 [astro-ph/0703132].

[9] Hamamatsu Photonics K.K., Stabilized light sources for photomultiplier tubes, (2012) http: //www.hamamatsu.com/resources/pdf/etd/L11416\_L11494\_TACC1057E.pdf.

[10] D. Heck, J. Knapp, J.N. Capdevielle, G. Schatz, T. Thouw, CORSIKA: A Monte Carlo code to simulate extensive air showers. TechReport, (1998).

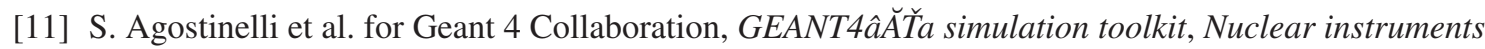
and methods in physics research section A: Accelerators, Spectrometers, Detectors and Associated Equipment 506 (2003) 250.

[12] J. Allison et al., Geant4 developments and applications, IEEE Transactions on Nuclear Science $\mathbf{5 3}$ (2006) 270.

[13] J. Allison et al., Recent developments in Geant4, Nuclear Instruments and Methods in Physics Research Section A: Accelerators, Spectrometers, Detectors and Associated Equipment 835 (2016) 186. 